\title{
$\beta$-Aminobutyric Acid-Induced Resistance Against Downy Mildew in Grapevine Acts Through the Potentiation of Callose Formation and Jasmonic Acid Signaling
}

\author{
Mollah Md. Hamiduzzaman, Gabor Jakab, Laurent Barnavon, Jean-Marc Neuhaus, and \\ Brigitte Mauch-Mani \\ University of Neuchâtel, Institute of Botany, Department of Biochemistry, Rue Emile-Argand 11, Case Postale 2, \\ $\mathrm{CH}-2007$ Neuchâtel, Switzerland
}

Submitted 31 January 2005. Accepted 13 April 2005.

\begin{abstract}
$\beta$-Aminobutyric acid (BABA) was used to induce resistance in grapevine (Vitis vinifera) against downy mildew (Plasmopara viticola). This led to a strong reduction of mycelial growth and sporulation in the susceptible cv. Chasselas. Comparing different inducers, the best protection was achieved with BABA followed by jasmonic acid (JA), whereas benzo $(1,2,3)$-thiadiazole-7-carbothionic acid- $S$ methyl ester (a salicylic acid [SA] analog) and abscisic acid (ABA) treatment did not increase the resistance significantly. Marker genes for the SA and JA pathways showed potentiated expression patterns in BABA-treated plants following infection. The callose synthesis inhibitor 2-deoxyD-glucose partially suppressed BABA- and JA-induced resistance against $P$. viticola in Chasselas. Application of the phenylalanine ammonia lyase inhibitor 2-aminoindan-2phosphonic acid and the lipoxygenase (LOX) inhibitor 5, 8, 11, 14-eicosatetraynoic acid (ETYA) also led to a reduction of $B A B A$-induced resistance (BABA-IR), suggesting that callose deposition as well as defense mechanisms depending on phenylpropanoids and the JA pathways all contribute to BABA-IR. The similar phenotype of BABA- and JAinduced resistance, the potentiated expression pattern of JA-regulated genes ( $L O X-9$ and $P R-4)$ following BABA treatment, and the suppression of BABA-IR with ETYA suggest an involvement of the JA pathway in BABA-IR of grapevine leading to a primed deposition of callose and lignin around the infection sites.
\end{abstract}

Additional keyword: oomycete.

Grapevine (Vitis vinifera), the major fruit crop worldwide, is affected by many diseases. Downy mildew, caused by the oomycete Plasmopara viticola, especially leads to massive damage and yield losses in this crop if no protective measures are taken. Although some grape cultivars (e.g., Solaris and Regent)

Corresponding author: B. Mauch-Mani; Fax: +41 32718 2201; E-mail: brigitte.mauch@unine.ch

Current address of L. Barnavon: INRA Dijon, Université de Bourgogne, 17, rue Sully, BP 86510, 21065 Dijon CEDEX, France.

Nucleotide sequence data is available in the GenBank database under accession numbers CF074675 for PR-1, CF074510 for PR-4, and AY159556 for LOX-9. are resistant against downy mildew, most traditional cultivars, planted on over $90 \%$ of the wine-growing area, are susceptible to this disease, necessitating an intensive use of plant protection chemicals to limit the damage in the vineyards. Because this is not compatible with a modern sustainable viticulture which aims at lowering the input of pesticides into the environment, and in view of the appearance of pesticide-resistant pathogen strains, efforts are being made to develop alternative protection strategies. One solution might be the activation of the plant's own defense system, known as induced resistance (Sticher et al. 1997). Synthetic molecules such as salicylic acid (SA), 2,6-dichloro-isonicotinic acid (INA), and benzo (1,2,3)thiadiazole-7-carbothionic acid-S-methyl ester (BTH) are able to induce systemic acquired resistance (SAR) in a variety of plants against a wide range of microbial pathogens (Sticher et al. 1997). This type of induced resistance is characterized by various early cellular changes and an increase in SA synthesis in both primary infected and distally noninfected leaves. Moreover, SAR is typified by enhanced systemic expression of socalled SAR genes coding for pathogenesis-related (PR) proteins (Ryals et al. 1996). Some of these proteins have demonstrated antifungal activity (Mauch et al. 1988); therefore, it is thought that they contribute to protection and they commonly are used as markers for SAR. SAR normally requires a lapse period between treatment and inoculation (Kessmann et al. 1994), allowing for signal transduction and gene activation to take place. This is the case with SAR induction by either biological agents (e.g., Tobacco mosaic virus in Nicotiana spp.) or chemical activators (e.g., SA, INA, and BTH).

Differential expression of chitinases has been used as a characteristic factor to evaluate the SAR response in grapevine against $P$. viticola by Busam and colleagues (1997b). These authors also implied that the expression of genes of the phenylpropanoid pathway in grapevine was induced by SAR activetors and suggested a role for $S$-adenosyl-L-methionine:transcaffeoyl-coenzyme A 3-O-methyltransferase (CCoAOMT) and stilbene synthase in the disease-resistance response (Busam et al. 1997a).

Jasmonic acid (JA), a product of the lipoxygenase (LOX) pathway, has been proposed to be a signal transducer of defense reactions in plants. Methyl jasmonate (MJ), for example, has been shown to induce accumulation of proteinase inhibitors in tobacco cells (Rickauer et al. 1992) and LOXs are induced in various plants following pathogen attack (Fournier et al. 1993; Melan et al. 1993). Farmer and associates (2003) proposed the 
existence of two pathways by which JA-related compounds can activate genes: both cyclopentenones, such as oxophytodienoic acid (OPDA), and JA activate and repress the expression of different, but overlapping sets of genes.

Another type of resistance, known as induced systemic resistance (ISR), mediated by nonpathogenic, root-colonizing pseudomonads, has been observed in several plant species (Duijff et al. 1998; Van Peer et al. 1991) and shown to induce resistance in all parts of the plant (Van Loon et al. 1998). Interestingly, ISR functions independently of SA and $P R$ gene activation, but requires components of the JA and ethylene responses (Pieterse et al. 1998). Indeed, jarl (Staswick et al. 1992) and etrl (Bleecker et al. 1988) mutants do not express ISR upon treatment with pseudomonads (Pieterse et al. 1998). Thus, SAR and ISR are regulated by distinct signaling pathways.

The nonprotein amino acid $\beta$-aminobutyric acid (BABA) previously has been shown to induce resistance against many different oomycetes. Local treatments of tomato, potato, and tobacco with BABA led to a systemic protection against Phytophthora infestans and Peronospora tabacina, respectively (Cohen 1994; Cohen et al. 1994). BABA also was effective in inducing resistance against downy mildew in lettuce (Pajot et al. 2001), cauliflower (Silue et al. 2002), and Arabidopsis (Zimmerli et al. 2000). The protective effect of BABA is not restricted to oomycetes because plants also have been shown
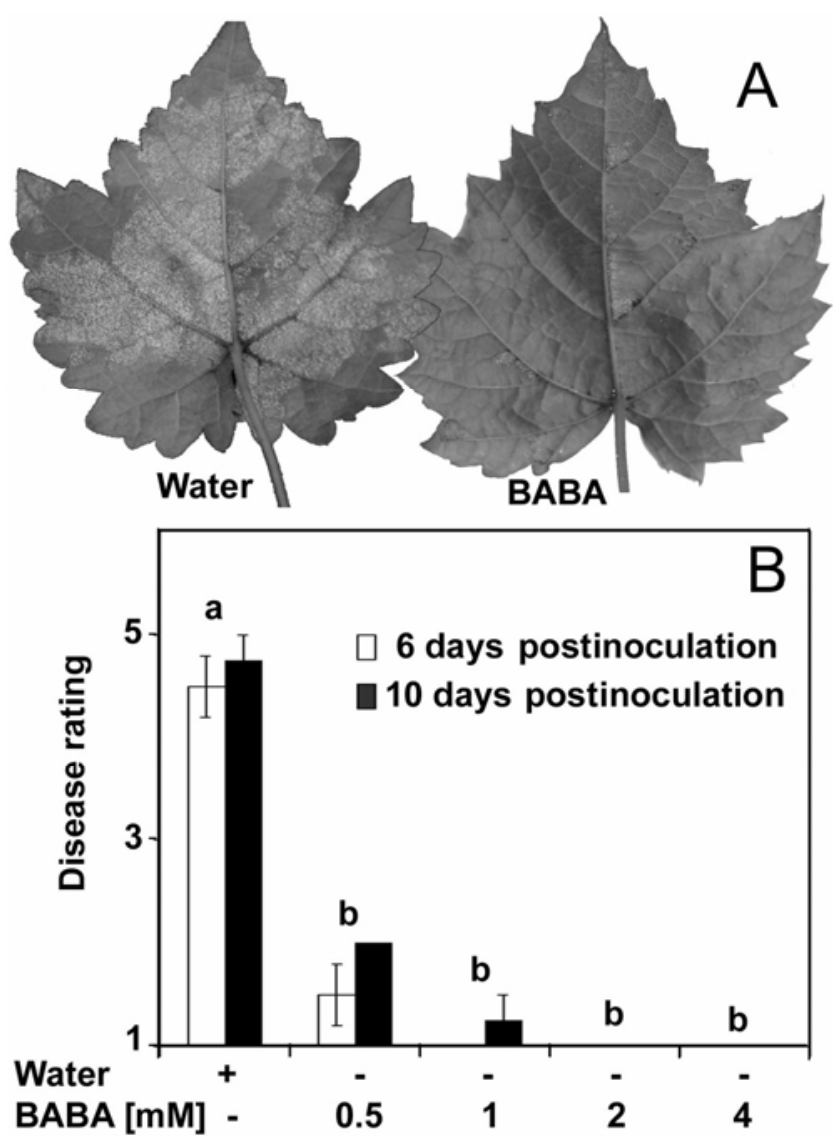

Fig. 1. Development of downy mildew (Plasmopara viticola) on seedlings of grapevine (Chasselas). A, Sporulation of $P$. viticola on the leaves after 6 days of inoculation $(0.5 \mathrm{mM} \beta$-aminobutyric acid [BABA]). B, Disease rating at 6 days (white bars) and 10 days (dark bars) post inoculation. Seedlings of grapevine (Chasselas) were taken at the four- to five-leaf stage. The plants were soil-drenched with water or BABA $(0.5,1,2$, or 4 $\mathrm{mM}) 2$ days prior to challenge inoculation with sporangia of $P$. viticola (5 $\times 10^{4}$ sporangia $\mathrm{ml}^{-1}$ ). Values presented are means \pm standard error of the mean (Tamhane's test, $\alpha=0.05$ ). The experiment was repeated three times with similar results. to build up resistance toward fungi, nematodes, and viruses following BABA treatment (Cohen et al. 2002; Jakab et al. 2001). Cohen and associates (1999) observed that BABA protected grape leaves from mildew infection when applied post infectionally. It has been speculated that BABA deteriorates penetrated host cells so that translocation of nutrients into the haustoria is blocked, thus prohibiting further mycelial growth and sporangial production (Steiner and Schönbeck 1997). A direct antimicrobial activity of this chemical has never been observed. Experiments with ${ }^{14} \mathrm{C}$-labeled BABA clearly show that it is not metabolized in tomato (Cohen and Gisi 1994) or in Arabidopsis (Jakab et al., 2001), ruling out the involvement of a BABA metabolite acting as an antimicrobial compound in the plant. Thus, BABA-mediated resistance is most likely based on the activation of host resistance mechanisms. Some studies report an induction of $P R$ genes after BABA treatment (Cohen et al. 1994), whereas others state the contrary (Cohen 1994). Zimmerli and associates (2000) observed that the protective effect of BABA in Arabidopsis was due to the potentiation of natural defense mechanisms against biotic stresses, a phenomenon later referred to as priming (Conrath et al. 2002).

Priming is the capacity of a plant to express a faster and stronger basal defense response upon pathogen infection (Conrath et al. 2002). BABA-primed resistance of Arabidopsis against Hyaloperonospora parasitica correlated with an earlier and stronger formation of callose-rich papillae (Zimmerli et al. 2000); therefore, it suggested that, in this case, induced resistance (BABA-IR) was based on primed callose deposition. The plant hormone abscisic acid (ABA) seems to play an important role in this priming reaction (Ton et al. 2005; Ton and MauchMani 2004). However, exogenous application of ABA prior to inoculation increases the susceptibility of tomato, Arabidopsis spp., and grapevine to various pathogens (Flors et al. 2005).

BABA treatment also potentiated the SA-regulated defense mechanisms in Arabidopsis (Zimmerli et al. 2000), contributing to the observed BABA-IR against $H$. parasitica. Mutants of Arabidopsis that are impaired in priming through BABA also are impaired in the expression of induced resistance (Ton et al. 2005). This clearly shows that BABA-IR has a genetic basis. Recently, Aziz and associates (2003) reported that the $\beta$ 1,3-glucan laminarin was an efficient elicitor of defense response in grapevine cells and plants against Botrytis cinerea and Plasmopara viticola and that it acted through priming.

To investigate the components playing a role in the establishment of BABA-IR against $P$. viticola in grapevine, different staining techniques were used to visualize biochemical changes at the cellular level. The reactions observed during BABA-IR in the susceptible cultivar were compared with naturally occurring disease resistance in the resistant cultivar. The mechanisms and the involvement of different signal transduction pathways in BABA-IR against $P$. viticola were investigated. Because signaling mutants of grapevine are not available, different metabolic inhibitors were used to achieve this goal.

\section{RESULTS}

BABA-treated grapevine plants show resistance against $P$. viticola and primed PR gene expression.

Seedlings of grapevine cv. Chasselas were treated by soil drench with either water (control) or BABA $(0.5 \mathrm{mM})$. The inoculation with $P$. viticola was performed by dipping the seedlings in a sporangial suspension of the oomycete. At 6 days postinoculation (dpi), heavy sporulation of downy mildew was observed on the abaxial surface of the inoculated leaves of the control plants (Fig. 1A). BABA treatment, however, highly reduced the sporulation of $P$. viticola into restricted patches on the lower leaf surface (Fig. 1A). Using different concentrations 
of BABA, the disease severity was quantified at 6 and $10 \mathrm{dpi}$. White clusters of sporangiophores were observed on the lower surface of the inoculated leaves, covering almost $100 \%$ of the leaf surface of nontreated (control) seedlings 6 days after inoculation. At the same time point, sporulation was absent in plants treated with 1,2 , or $4 \mathrm{mM}$ BABA, whereas sparse sporulation (10 to $20 \%$ of leaf surface) was observed in plants treated with the lowest concentration of BABA $(0.5 \mathrm{mM})$ (Fig. 1B). A slight increase in sporulation 10 days after inoculation was observed in plants treated with 0.5 or $1 \mathrm{mM}$ BABA, whereas 2 and $4 \mathrm{mM}$ BABA still gave full protection (Fig. 1B). Light necrosis was observed in leaves of BABA-treated plants upon infection with $P$. viticola.

Expression profiling of marker genes for both the SA and JA signal transduction pathways was used to determine their involvement in the observed BABA-IR. No appropriate marker genes of grapevine have been characterized; therefore, we determined the expression pattern of several defense-related genes after treatment with JA, BTH, and ABA. $P R-1$ showed a high induction after BTH treatment, although it also responded to other treatments and stress conditions, but to a lower extent (Fig. 2A). In contrast, $P R-4$ and $L O X-9$ were highly induced by JA treatment (Fig. 2A). Therefore, $P R-1$ was selected as a marker gene for the SA pathway and $P R-4$ and $L O X-9$ as marker genes for JA signaling. All three marker genes of grapevine showed a potentiated expression pattern in BABA-treated plants after infection with $P$. viticola (Fig. 2B). The expression level of $P R-1$ reached its maximum $24 \mathrm{~h}$ after inoculation in the controls, but $13 \mathrm{~h}$ earlier in BABA-treated seedlings. Both $P R-4$ and $L O X-9$ showed a potentiated expression pattern in BABAtreated plants with an increase in their expression $7 \mathrm{~h}$ postinoculation (hpi) and peaking at $11 \mathrm{hpi}$ (Fig. 2B).

\section{Development of $P$. viticola in susceptible}

and resistant grapevine with and without $B A B A$ treatment.

To better understand the mechanisms of BABA-induced resistance in grapevine, in addition to the highly susceptible cv. Chasselas, the resistant cv. Solaris was included as a reference. Discs punched from different leaves were placed with their adaxial surface on wet Whatman paper in petri dishes. The leaf disks were inoculated with droplets of a sporangial suspension of $P$. viticola. The sporangia released zoospores that germinated and the germ tubes penetrated into the stomata approximately 7 hpi. White sporulation of $P$. viticola could be observed by naked eye 4 days after inoculation in Chasselas (Fig. 3A). The presence of clusters of sporangiophores was confirmed with a dissecting microscope. The density of sporangiophores was very high in Chasselas and no necrosis of the plant tissue was observed (Fig. 3A). However, only very few sporangiophores appeared on BABA-treated Chasselas (Fig. 3B) and necrosis surrounded the inoculation site. In Solaris, a few sporangiophores developed occasionally and necrosis surrounded the inoculation site similarly to BABAtreated Chasselas (Fig. 3C). Sporulation was completely absent in BABA-treated Solaris and leaf disks showed a brown discoloration at the cut edge (Fig. 3D).

At the microscopic level, hyphal growth of $P$. viticola was observed at $7 \mathrm{hpi}$ and spread within 3 days after inoculation in untreated Chasselas (Fig. 3E, control). In BABA-treated Chasselas, however, necrotic plant cells were observed adjacent to the hyphae (Fig. 3F). At $5 \mathrm{dpi}$, sporulation was observed in nontreated Chasselas. Several sporangiophores grew out of a single stoma in nontreated disks (Fig. 3G) but only single, distorted sporangiophores bearing a low number of sporangia grew out from single stomata following BABA treatment (Fig. $3 \mathrm{H})$ and this weak sporulation was not visible macroscopically in the BABA-treated disks. Sporangiophores usually were ab- sent in Solaris. Occasionally, sparse sporulation was observed in nontreated Solaris but never following BABA treatment.

\section{Quantification of callose and lignin deposition} around the infection sites.

Plant tissues stained with calcofluor aniline blue display a bright yellow-white fluorescence under UV light that corresponds to callose deposition. This fluorescence was absent in nontreated Chasselas (Fig. 3I) but could be observed in BABA-treated Chasselas (Fig. 3J) as well as in both treated (Fig. 3K) and nontreated Solaris (Fig. 3L). The fluorescence was much stronger 3 days after inoculation than after 1 day, and it was stronger in treated than nontreated Solaris plants. The light blue-white fluorescence, clearly distinguishable from
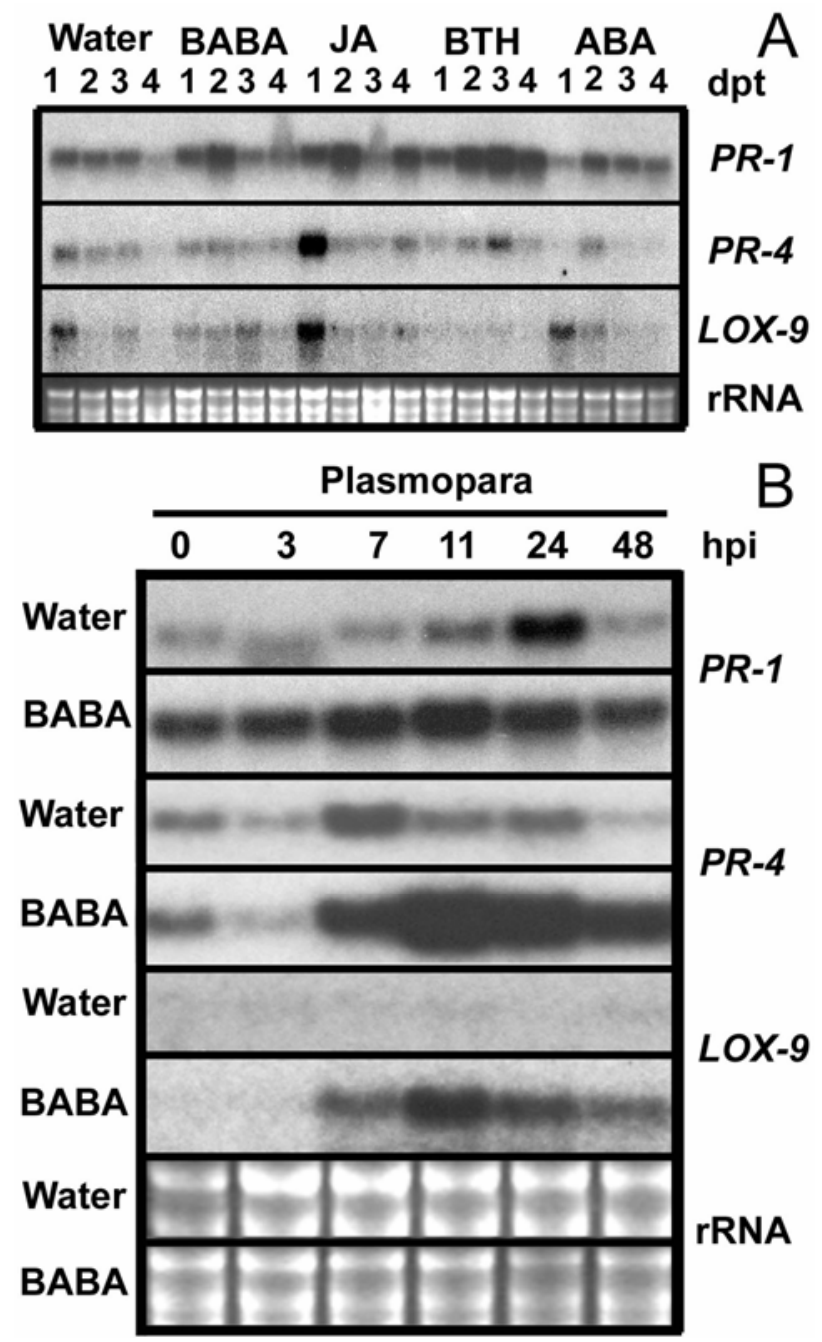

Fig. 2. Expression pattern of the $P R-1, L O X-9$ and $P R-4$ genes in seedlings of grapevine (Chasselas) upon $\mathbf{A}$, treatment with different inducers and $\mathbf{B}$, infection with Plasmopara viticola. A, Inducers ( $\beta$-aminobutyric acid [BABA], $1 \mathrm{mM}$; jasmonic acid [JA], $50 \mu \mathrm{M}$; benzo (1,2,3)-thiadiazole-7carbothionic acid-S-methyl ester [BTH], $300 \mu \mathrm{M}$; and abscisic acid [ABA], $40 \mu \mathrm{M}$ ) were applied as soil drench and leaves of seedlings were collected at different time points as indicated. B, Seedlings were soildrenched with water or BABA $(1 \mathrm{mM}) 2$ days prior to challenge inoculation with sporangia of $P$. viticola $\left(5 \times 10^{4}\right.$ sporangia $\left.\mathrm{ml}^{-1}\right)$. Inoculated leaves of seedlings were collected at different time points as indicated. Total RNA was extracted at different time points after infection. Each time point represents $1 \mathrm{~g}$ of leaf materials (eight leaves from four different plants). RNA blots were hybridized with $P R-1, P R-4$ and $L O X-9$ probes. Ethidium bromide staining of the RNA gel (rRNA) was used to show equal loading. The experiment was repeated twice with similar results; dpt: days post treatment; hpi: hours post inoculation. 

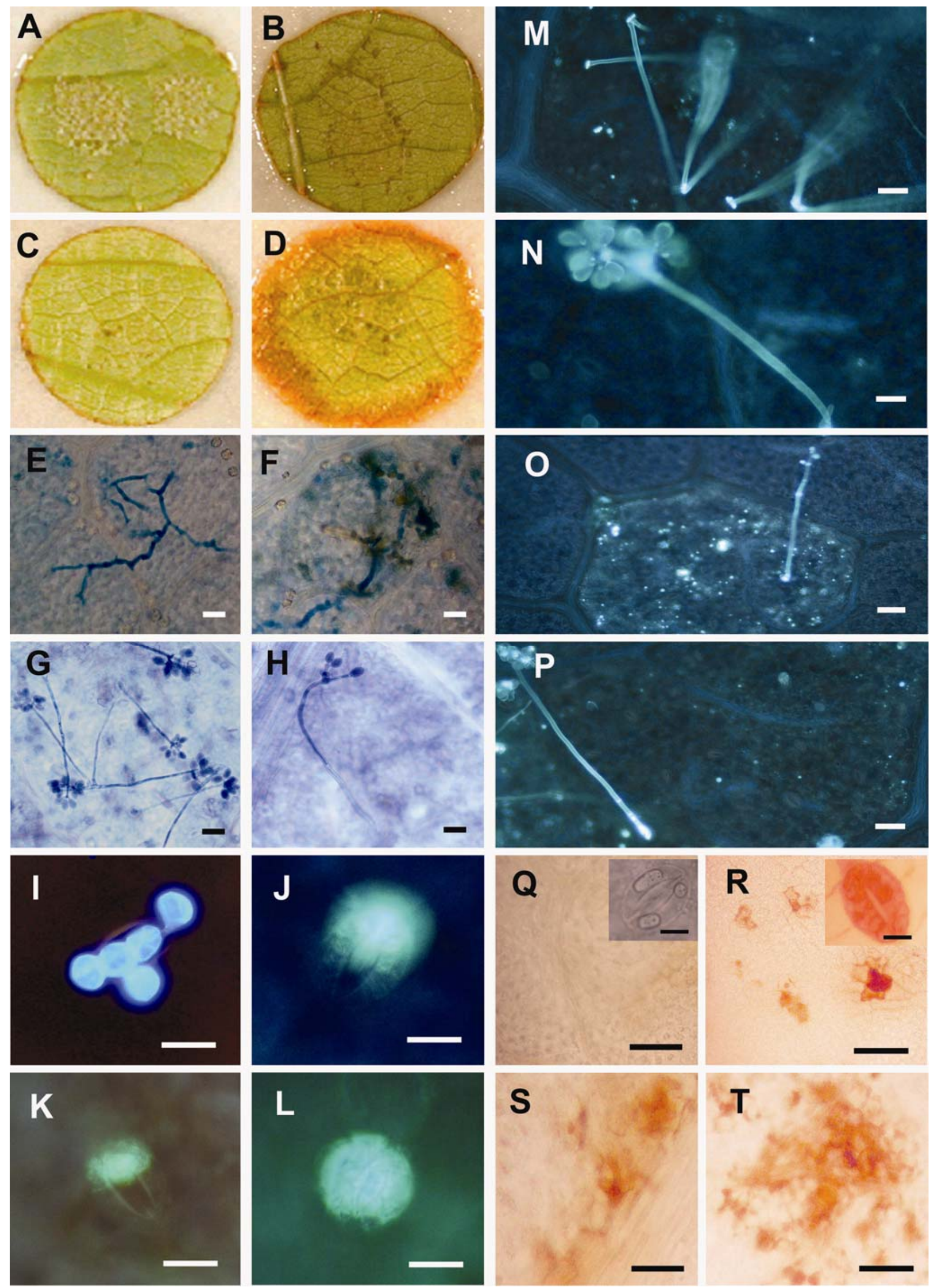
the yellow-white fluorescence of callose, shows the presence of oomycete structures on the leaves (Fig. 3M). Strong yellow-white fluorescence was observed mostly in stomata and in the host cells surrounding the sporangiophores in BABAtreated Chasselas as well as in both nontreated and BABAtreated Solaris. No callose deposition was visible in nontreated Chasselas within 3 days of inoculation, although light yellow-white fluorescence sometimes appeared in the veins.

An inhibitor of callose synthesis, 2-deoxy-D-glucose (DDG) (Jakab et al. 2001), was used to demonstrate the correlation between callose deposition and the observed yellow-white fluorescence in grapevine leaves. Yellow spots corresponding to callose deposition were absent in nontreated Chasselas (Fig. $3 \mathrm{M}$ ) as well as in DDG-treated plants (Fig. $3 \mathrm{~N}$ ), whereas they were observed in high density surrounding the infected area in BABA-treated Chasselas (Fig. 30). The callose deposition density, however, decreased with the co-application of DDG and BABA (Fig. 3P).

Lignification in grapevine leaves was visualized by phloroglucinol-HCl staining leading to a red coloration of lignin. Lignification was first observed $24 \mathrm{hpi}$ and it increased with time. In nontreated Chasselas, no red-stained cells were observed after infection with P. viticola (Fig. 3Q). However, in BABA-treated Chasselas and in both nontreated and BABAtreated Solaris, red-stained cells (cell walls and cytoplasm) were observed, mostly confined to the host cells surrounding the infected area (Fig. 3R, S, and T, respectively) and in the guard cells of stomata (Fig. 3R, inset).

\section{Comparison of the efficiency \\ of different inducers of resistance against $P$. viticola.}

To compare BABA-IR with BTH- and JA-induced resistance, leaf disks of plants were treated with the respective substances, inoculated and the number of sporangia were counted at $6 \mathrm{dpi}$. The highest number of sporangia was observed in nontreated control plants (Fig. 4A; Chasselas). BABA induced highest protection against $P$. viticola, followed by JA, whereas BTH treatment led to very low protection. Some phytotoxic effect (necrosis at the cut edges of leaf disks) was observed upon treatment with the highest concentrations of BTH and JA. Therefore, care was taken to use inducer concentrations below toxic levels because necrosis could interfere with biotrophic growth of $P$. viticola. Compared with the seedling assays (Fig. 2B), the expression of marker genes in leaf disks during BABA-IR against $P$. viticola infection also was analyzed. $P R-1$ was highly expressed in both water- and BABA-treated leaf disks, whereas $P R-4$ and $L O X-9$ showed potentiated expression patterns in BABA-treated leaf disks upon infection with $P$. viticola (Fig. 4B). The potentiated expression of the JA pathway was observed in BABA-treated leaf disks with a peak at 7 hpi which was similar to the seedling assay.

\section{The role of callose in resistance against $P$. viticola.}

The callose synthesis inhibitor DDG also was used to assess the importance of callose in both genetic and BABA-induced resistance. Leaf disks were treated and inoculated with $P$. viticola, and the number of sporangia per leaf disk was counted 6 dpi (further described below). In the case of the susceptible cv. Chasselas, the number of sporangia was highest in nontreated control plants as well as in DDG-treated plants (Fig. 5A), whereas the lowest number of sporangia was observed in BABA-treated plants (Fig. 5A). The number of sporangia increased with increasing concentrations of DDG in BABAtreated plants (data not shown). To investigate the relationship between callose deposition and sporulation, callose deposition was quantified in duplicate experiments with the same treatments. The highest callose deposition, measured as intensity of yellow in micrographs, was observed in BABA-treated Chasselas (Fig. 5B). The observed callose intensity decreased with the co-application of DDG (Fig. 5B). Solaris displays genetically determined resistance against $P$. viticola; however, the number of sporangia increased significantly in DDG-treated infected Solaris plants (Fig. 5C). The number of sporangia was inversely correlated with callose deposition, indicating the involvement of callose in resistance against $P$. viticola (Fig. 5D).

The plant hormone ABA recently has been shown to play a role in BABA-regulated callose deposition in Arabidopsis (Ton and Mauch-Mani 2004); therefore, the potential role of ABA in defense of grapevine was investigated. Leaf disks were treated and inoculated with $P$. viticola, and the number of sporangia per leaf disk was counted at 6 dpi. There was no significant level of resistance in ABA-treated plants at lower concentrations, whereas a small reduction in the number of sporangia was observed with a high concentration of $\mathrm{ABA}$ (Fig. 5E). Minor phytotoxic effects were observed in leaf veins (brownish color) upon the treatment with higher concentration of ABA. Interestingly, the level of resistance against $P$. viticola in ABA-treated plants was significantly lower than in JAtreated plants (Fig. 5E). To investigate the relationship between callose intensity and formation of sporangia in JAinduced resistance, callose deposition was quantified in the same experiment. The highest callose intensity was observed in JA-treated Chasselas and it decreased with the coapplication of DDG. Callose intensity was much lower in ABAtreated plants than JA-treated plants (Fig. 5F).

Fig. 3. Development of necrosis, priming of callose deposition, and lignification in $\beta$-aminobutyric acid (BABA -treated grapevine following infection with Plasmopara viticola. A through T, Leaf disks (1 cm in diameter) of grapevine (A, B, E-J, and M-R are Chasselas; C, D, K, L, S, and T are Solaris) were punched from the third and fourth leaves from the top of a plant. Discs were treated with water and BABA (1 mM) $24 \mathrm{~h}$ prior to challenge inoculation with sporangia of $P$. viticola $\left(5 \times 10^{4}\right.$ sporangia $\left.\mathrm{ml}^{-1}\right)$. The pictures were taken 5 days post inoculation (dpi) with $P$. viticola (A, B, C, and D). Leaf disks were stained with lactophenol-trypan blue and analyzed by light microscopy (E, F, G, and H), stained with calcofluor-aniline blue and analyzed by epifluorescence microscopy (I, J, K, L, M, N, O, and P), or stained with phloroglucinol-HCL and analyzed by light microscopy (Q, R, S, and T). A, Nontreated control with white sporulation (Chasselas); B, BABA-treated Chasselas showing light necrosis at the inoculation site; C, nontreated Solaris; D, BABA-treated Solaris displaying light necrosis at the inoculation site and an increased necrotic wound reaction at the edge of the disk. E, intercellular hyphae of $P$. viticola in nontreated control (3 dpi); F, intercellular hyphae surrounded by necrosis in BABA-treated leaf tissue (3 dpi); G, cluster of sporangiophores (6 dpi) on nontreated control; $\mathbf{H}$, distorted sporangiophore (6 dpi) with low number of sporangia following BABA treatment; I, nontreated control Chasselas, germinating zoospores ( 2 dpi) entering into a stoma (no callose deposition); J, BABA-treated Chasselas, callose deposition in the stomatal cavity, K, nontreated Solaris, callose deposition in the stomatal cavity; L, BABA-treated Solaris, prominent callose deposition in the stomatal cavity. Chasselas ( 5 dpi); $\mathbf{M}$, nontreated control with numerous sporangiophore stalks visible; N, callose inhibitor 2-deoxy-D-glucose (DDG) treated, development of sporangiophores (no callose deposition); O, BABA treated, callose deposition visible as yellow-white spots surrounding a single, distorted sporangiophore; P BABA and DDG treated, decreased callose deposition visible as lower number of yellow-white spots; Q, nontreated control Chasselas, (3 dpi) no lignification (redcolored tissues) visible; and R, BABA-treated Chasselas, 3 dpi, red-stained lignin in tissues and in stomatal cells (inset). S, Nontreated Solaris and T, BABAtreated Solaris, both showing lignified cells (stained red) at $3 \mathrm{dpi}$. Bar $=20 \mu \mathrm{m}$. 
The role of the phenylpropanoid pathway in BABA-IR against $P$. viticola.

To further clarify the involvement of phenylpropanoid pathway-derived defense responses in BABA-IR of grapevine, the effect of the phenylalanine ammonia lyase (PAL) inhibitor 2aminoindan-2-phosphonic acid (AIP) (Zon and Amrhein 1992) and the callose inhibitor DDG together with BABA on the infection of $P$. viticola was analyzed (Fig. 6). Leaf disks were treated and inoculated, and the number of sporangia per leaf disk was determined at $6 \mathrm{dpi}$. In Chasselas, the number of sporangia was highest in nontreated control plants (Fig. 6A).
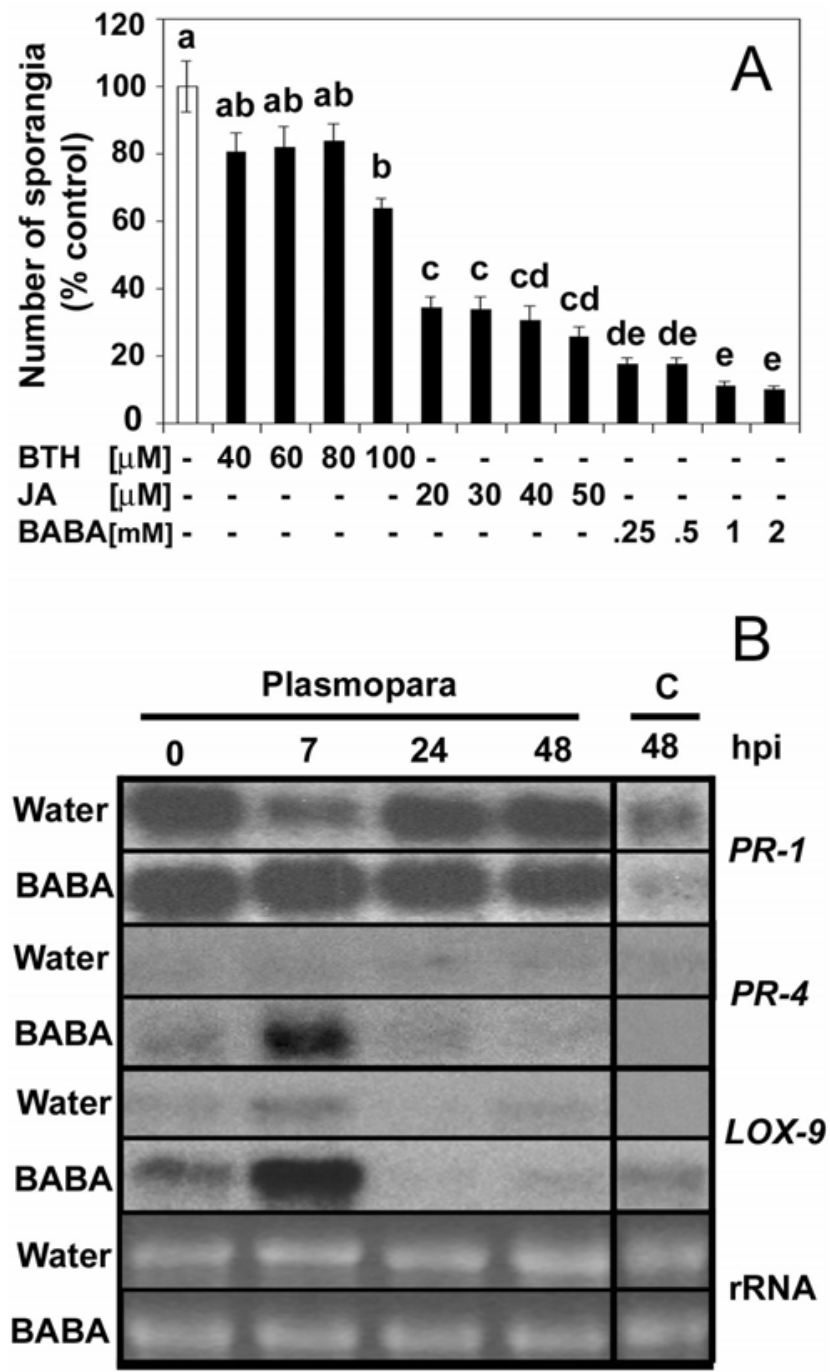

Fig. 4. Effect of benzo (1,2,3)-thiadiazole-7-carbothionic acid-S-methyl ester (BTH), jasmonic acid (JA), and $\beta$-aminobutyric acid (BABA) on the infection of Plasmopara viticola in grapevine (Chasselas) and expression pattern of marker genes in leaf disks after infection. A, Leaf disks (16 disks/treatment, 4 disks/replication, four replications/treatment) were punched from the third and fourth leaves from the top of a plant. They were treated with water (white bar), and BTH, JA, and BABA (dark bars) $24 \mathrm{~h}$ prior to challenge inoculation with sporangia of $P$. viticola $\left(5 \times 10^{4}\right.$ sporangia $\left.\mathrm{ml}^{-1}\right)$. The number of sporangia $(P$. viticola $)$ per leaf disk was determined 6 days after inoculation. Values presented are means \pm standard error of the mean. Different letters indicate statistically significant differences (Tamhane's test, $\alpha=0.05$ ). The experiment was repeated three times with similar results. B, Leaf disks were treated with water or BABA $(1 \mathrm{mM}) 24 \mathrm{~h}$ prior to challenge inoculation with sporangia of $P$. viticola $(5 \times$ $10^{4}$ sporangia ml-1). Inoculated disks were collected at different time points as indicated. Each time point represents 16 leaf disks. RNA blots were hybridized with $P R-1, P R-4$, and $L O X-9$ probes. Ethidium bromide staining of the RNA gel (rRNA) was used to show equal loading. The experiment was repeated twice with similar results; hpi: hours post inoculation.
There was no significant difference between nontreated control plants and plants treated either with AIP alone or with AIP together with DDG, demonstrating that these treatments had no negative effect on $P$. viticola. In contrast, BABA-treated plants showed the lowest number of sporangia (Fig 6A). A small increase in the number of sporangia was observed with the coapplication of AIP with BABA, and this coincided with a reduced level of lignification (data not shown). However, the number of sporangia increased significantly when DDG was applied together with AIP in BABA-treated Chasselas. In the resistant cv. Solaris, AIP alone has no significant effect on the sporulation of $P$. viticola compared with nontreated plants (Fig. 6B). The number of sporangia increased significantly in plants treated with AIP and DDG together. BABA-treated plants showed the highest protection against $P$. viticola. The strong BABA-IR in Solaris was not suppressed with DDG and AIP treatment (Fig. 6B).

\section{The role of JA-dependent defenses}

in BABA-IR against $P$. viticola.

To identify the role of the JA-defense signaling pathway in BABA-IR, the LOX inhibitor 5, 8, 11, 14-eicosatetraynoic acid (ETYA) was used. In Chasselas, the number of sporangia was as high in ETYA-treated as in nontreated plants (Fig. 6C), indicating that ETYA has no direct effect on $P$. viticola. The low number of sporangia in BABA-treated plants increased with the co-application of ETYA but remained lower than in control plants. In Solaris, the number of sporangia increased

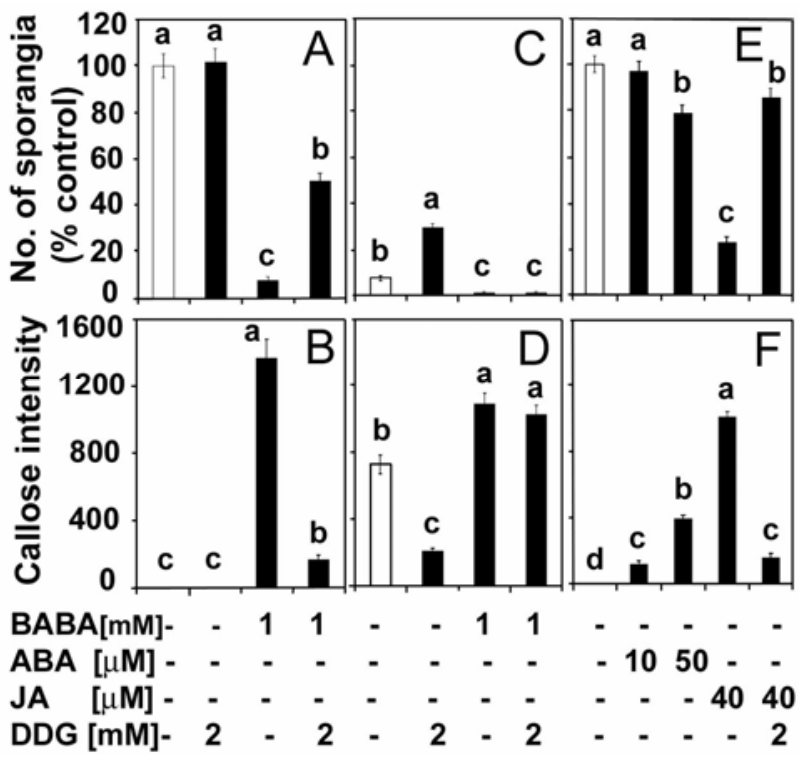

Fig. 5. Effect of different inducers and the callose synthesis inhibitor 2 deoxy-D-glucose (DDG) on the infection of grapevine by Plasmopara viticola and on the intensity of callose deposition in grapevine (Chasselas). Leaf disks (16 disks/treatment, 4 disks/replication, four replications/treatment) were punched from the third and fourth leaves from the top of a plant. Leaf disks were treated with water (white bars); $\mathbf{A}, \mathbf{B}$, $\mathbf{C}$, and $\mathbf{D}, \beta$-aminobutyric acid (BABA), $\mathbf{E}$ and $\mathbf{F}$, abscisic acid (ABA) or jasmonic acid (JA); or DDG $24 \mathrm{~h}$ prior to challenge inoculation with sporangia of $P$. viticola $\left(5 \times 10^{4}\right.$ sporangia $\left.\mathrm{ml}^{-1}\right)$ as indicated below the figure. A and E, Number of sporangia ( $P$. viticola) per leaf disk (Chasselas) 6 days after inoculation. $\mathbf{B}$ and $\mathbf{F}$, Callose intensity in leaf disks of Chasselas (3 dpi). C, Number of sporangia ( $P$. viticola) per leaf disk (Solaris) 6 days after inoculation. D, Callose intensity in leaf disks of Solaris ( $3 \mathrm{dpi})$. Callose quantification was performed by determining the number of fluorescent pixels corresponding to callose deposition on digital photographs of the infected leaf area. Values presented are means \pm standard error of the mean. Different letters indicate statistically significant differences (Tamhane's test, $\alpha=0.05$ ). The experiment was repeated three times with similar results. 
significantly with the application of ETYA (Fig. 6D). However, ETYA was not able to suppress the strong resistance in BABA-treated Solaris. To verify the inhibitory effect of ETYA on the JA pathway in grapevine leaf disks, the expression pattern of marker genes for this pathway was analyzed. Pretreatment with ETYA prior to BABA treatment strongly reduced the expression of both $P R-4$ and $L O X-9$ following infection with Plasmopara spp. (Fig. 7).

\section{DISCUSSION}

Our results confirm that BABA protects grapevine from infection by $P$. viticola (Cohen et al. 1999). BABA induced a significant level of resistance against $P$. viticola in grapevine seedlings (Chasselas) when applied as soil drench, because sparse sporulation was observed at 10 dpi with only the lowest concentration of BABA. This suggests that BABA translocated through the root system to protect the different plant parts and delayed the sporulation of $P$. viticola. These results confirmed earlier findings of Cohen and colleagues (1999) and showed that the interaction of Chasselas with downy mildew is amenable for further studies of BABA-IR in grapevine.

Leaf disk assays showed a big difference in the reactions of the two cultivars of grapevine following infection with downy mildew. The susceptible cultivar (Chasselas) developed early, large lesions (visible 3 to $4 \mathrm{dpi}$ ) with clusters of sporangiophores and conspicuous sporulation, whereas the resistant cultivar (Solaris) developed necrotic spots with late, sparse sporulation. The necrosis observed in BABA-treated Chasselas plants indi-

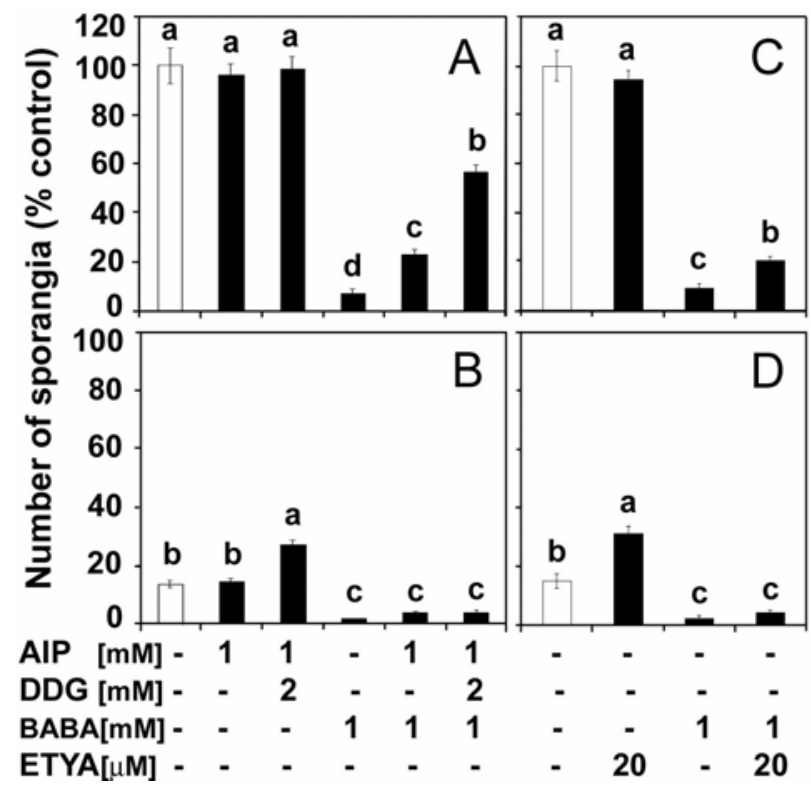

Fig. 6. Effect of the phenylalanine ammonia lyase (PAL) inhibitor 2-aminoindan-2-phosphonic acid (AIP), callose synthesis inhibitor 2-deoxy-Dglucose (DDG), and the lipoxygenase (LOX) inhibitor 5, 8, 11, 14-eicosatetraynoic acid (ETYA) together with $\beta$-aminobutyric acid (BABA) on the infection of Plasmopara viticola in grapevine. $\mathbf{A}$ and $\mathbf{C}$, Chasselas and B and D, Solaris (16 disks/treatment, 4disks/replication, four replications/treatment) leaf disks were punched from the third and fourth leaves from the top of a plant and. $\mathbf{A}$ and $\mathbf{B}$, Leaf disks were treated with water (white bars), and BABA, AIP, or DDG (dark bars) $24 \mathrm{~h}$ prior to challenge inoculation with sporangia of $P$. viticola $\left(5 \times 10^{4}\right.$ sporangia $\left.\mathrm{ml}^{-1}\right)$. $\mathbf{C}$ and D, The disks were first floated for $2 \mathrm{~h}$ on ETYA solution before BABA or water control treatment applied $24 \mathrm{~h}$ prior to challenge inoculation with sporangia of $P$. viticola $\left(5 \times 10^{4}\right.$ sporangia $\left.\mathrm{ml}^{-1}\right)$. Number of sporangia $(P$. viticola) per leaf disk after 6 days of inoculation. Values presented are means \pm standard error of the mean. Different letters indicate statistically significant differences (Tamhane's test, $\alpha=0.05$ ). The experiment was repeated three times with similar results. cates the accumulation of phenolic compounds. The single distorted sporangiophores bearing a low number of sporangia that were observed in BABA-treated Chasselas are reminiscent of the occasional sparse sporulation in the (nontreated) resistant cv. Solaris. Gindro and associates (2003) showed that, in Solaris, $P$. viticola cannot complete its life cycle. This probably is due to a very rapid defense response, including the deposition of callose-like material in the substomatal cavity that takes place as soon as germ tubes try to penetrate the stomata of this resistant cultivar.

Histochemical studies were carried out to examine the biochemical changes at the cellular level upon infection with $P$. viticola. Callose deposition and lignification at the cellular level could contribute to prevent the infection of $P$. viticola in BABA-treated plants. Flavonoids have been shown to play a key role in another resistant Vitis sp. (V. rotundifolia); in addition; resveratrol at an early stage and flavonoids and lignin at a later stage have been proposed to play a role in restricting the growth of $P$. viticola in intermediate resistant species ( $V$. rupestris) (Dai et al. 1995). In plants, a pretreatment with low concentrations of SA or BABA primes the cells to react more quickly and efficiently to subsequent elicitor treatment or pathogen attack (Conrath et al. 2002). The biochemical changes at the cellular level in BABA-treated grapevine plants occurred

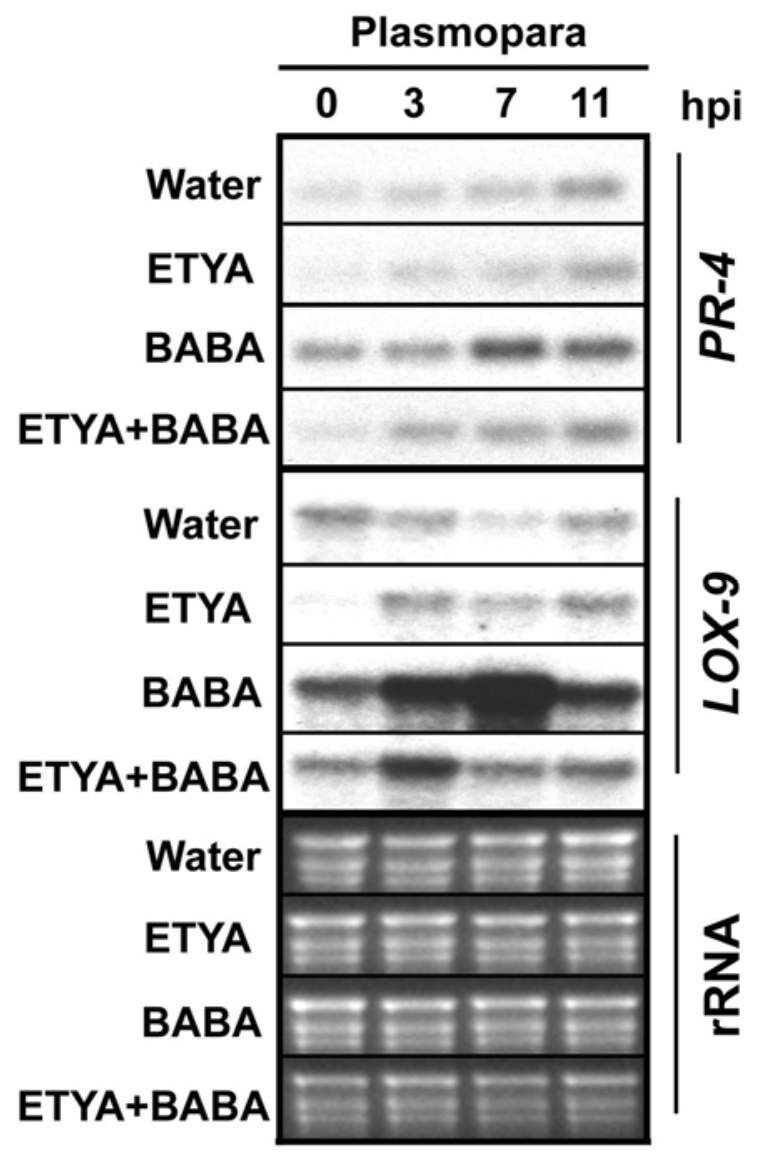

Fig. 7. Effect of the lipoxygenase (LOX) inhibitor 5, 8, 11, 14eicosatetraynoic acid (ETYA) on the expression pattern of jasmonic acidregulated marker genes. Leaf disks of Chasselas were first floated for $2 \mathrm{~h}$ on water (water and $\beta$-aminobutyric acid [BABA]) or ETYA solution (ETYA and ETYA + BABA) before BABA (BABA and ETYA + BABA) or water control (water and ETYA) treatment applied $24 \mathrm{~h}$ prior to challenge inoculation with sporangia of Plasmopara viticola $\left(5 \times 10^{4}\right.$ sporangia ml $\left.{ }^{1}\right)$. Inoculated disks were collected at different time points as indicated. Each time point represents 16 leaf disks. RNA blots were hybridized with $P R-4$ and $L O X-9$ probes. Ethidium bromide staining of the RNA gel (rRNA) was used to show equal loading; hpi: hours post inoculation. 
only upon infection with $P$. viticola; this suggests that here, too, BABA might act via priming. Biochemical changes were observed in epidermal, mesophyll, and guard cells and in cell walls of BABA-treated plants after inoculation with $P$. viticola. Callose deposition and lignification became visible as early as 24 hpi and increased during the following days. In the resistant cv. Solaris, callose deposition and lignification were more pronounced in BABA-treated than in nontreated plants. Because stomata are crucial for $P$. viticola infection, callose deposition and lignification in stomatal cells might restrict penetration and development of $P$. viticola in BABA-treated plants.

Application of the callose synthesis inhibitor DDG had no direct toxic effect on $P$. viticola; however, it suppressed BABA- and JA-induced resistance in Chasselas as well as basal resistance in Solaris. BABA- and JA-induced resistance positively correlated with intensity of callose deposition in Chasselas. Jakab and associates (2001) reported that the higher and faster accumulation of callose in ovules led to sterility of BABA-treated Arabidopsis flowers which could be reversed by a DDG treatment.

Callose deposition decreased with increasing concentrations of DDG and was inversely correlated with the number of sporangia. The highest callose intensity was observed in BABAtreated Solaris; however, DDG failed to completely suppress this strong resistance. BABA- and JA-induced resistance in Chasselas and basal resistance in Solaris were significantly reduced with DDG treatment, but this did not result in a susceptibility comparable with the one of nontreated Chasselas plants. This indicates that other factors besides callose deposition must be involved in the resistance process. Therefore, callose deposition is a major but not the only factor in BABA-IR against $P$. viticola.

The phenocopy hypersensitive-resistance lesions in BABAtreated grapevine infected by $P$. viticola have been shown to accumulate lignin-like deposits (Cohen et al. 1999). Callose accumulation has been observed in tolerant cultivars at later stages of infection, indicating that mechanisms different from callose deposition also are involved in defense against $P$. viticola (Kortekamp et al. 1997).

To elucidate further possible mechanisms contributing to BABA-IR against $P$. viticola, we examined the involvement of SA-, JA- and ABA-dependent defenses. A higher protection was observed against $P$. viticola in BABA- than in JA-treated plants and only a very limited effect could be observed with BTH and ABA. Callose accumulation was higher in BABAand JA-treated plants than in BTH- (data not shown) and ABA-treated plants. The number of sporangia in BABA- and JA-treated plants was inversely correlated with the intensity of callose deposition.

The lack of effects of ABA is in accordance with a report by Lu and associates (2001) describing the presence of higher ABA concentrations in grapevine shoots with symptoms of Pierce's disease than in healthy shoots. In tomato plants, the susceptibility to Botrytis cinerea was enhanced by ABA treatment (Audenaert et al. 2002). In contrast, ABA has been shown to regulate plant responses to abiotic stresses (Xiong et al. 2002) and to induce resistance against necrotrophic pathogens in Arabidopsis (Ton and Mauch-Mani 2004). The failure of the SA analog BTH to induce resistance against downy mildew of grape agrees with previous findings that BABA-IR in Arabidopsis against $H$. parasitica is SA independent (Zimmerli et al. 2000). The role of SA has been investigated in transgenic tobacco, which constitutively express the SA hydroxylase gene leading to a continuous transformation of SA into catechol (NahG) (Delaney 1997). BABA was found to be equally effective in NahG-expressing and wild-type Arabidopsis against downy mildew, suggesting that BABA may trigger a resistance-mediating signal transduction pathway downstream of SA (Zimmerli et al. 2000).

In grapevine, the JA pathway, rather than the SA and ABA signaling pathways, seems to be involved in induced resistance against downy mildew. Thomma and associates (1998) reported that the JA- and camalexin-insensitive mutants of Arabidopsis were susceptible to Alternaria brassicicola. In grapevine, the exogenous application of JA induced resistance not only to pathogens but also to foliar and root herbivores (Omer et al. 2000). In our experiments, BABA- and JA-induced resistance against $P$. viticola similarly were based on high callose deposition, indicating that callose deposition might depend on the JA defense signal pathway. In contrast, BABA-primed callose deposition in Arabidopsis spp. is based on ABA rather than JA signaling (Ton et al. 2005). A recent study of these two signaling pathways in Arabidopsis shows that they might have an antagonistic role in stress responses (Anderson et al. 2004). Therefore, further studies are needed to clarify the potential interactions of JA and ABA on callose deposition during BABA-IR in different plants.

The plant hormones SA and JA play key roles in the regulation of defense responses to different pathogens and herbivores. To investigate the involvement of signal transduction pathways in BABA-IR, the expression pattern of marker genes of the SA and JA pathways were tested in both seedling and leaf disk assays. In seedlings, the overall expression of $P R-1$ was low during downy mildew infection but an earlier and higher expression was observed following BABA treatment. This indicates that BABA is able to prime the SA signaling pathway in grapevine. However, the failure of BTH to induce resistance as well as the constitutively high expression of $P R-1$ in leaf disks show that, in this case, the SA pathway does not contribute to BABA-IR. On the other hand, the clear potentiated expression of $L O X-9$ and $P R-4$ in both BABA-treated seedlings and leaf disks following $P$. viticola infection further supports an involvement of the JA signal transduction pathway in BABA-IR. This is comparable with results that Arabidopsis mutants affected in JA biosynthesis or signaling are more susceptible to Pythium mastophorum (Vijayan et al. 1998) as well as to insect herbivory (McConn et al. 1997). A possible role for LOX-9in induced resistance in grapevine also has been proposed by Aziz and associates (2003). They showed that LOX-9 gene expression correlated with an increased defense following laminarin treatment of grapevine. Similarly, Aziz and associates (2004) reported that oligogalacturonides stimulated $P R$ gene activity which correlated with a reduced infection of grapevine plants by Botrytis cinerea.

In grapevine, it is not possible to assess the role of signaling pathways by using mutants in these pathways; therefore, the involvement of different signaling compounds were tested through biochemical inhibition of the pathways of interest.

The role of lignification and of the SA-dependent signaling pathway in BABA-IR was tested by applying the PAL inhibitor AIP. The resistance in BABA-treated Chasselas decreased only poorly with co-application of AIP, and AIP also failed to suppress the basal resistance in Solaris against Plasmopara viticola. However, BABA-IR in Chasselas and basal resistance in Solaris decreased significantly when plants were treated with both the callose inhibitor DDG and AIP. The synergistic effect of DDG and AIP on the resistance indicates that, in addition to callose deposition, other factors such as lignification might enhance the defense reaction against $P$. viticola. MauchMani and Slusarenko (1996) reported that PAL is involved in the synthesis of SA and precursors of lignification in Arabidopsis, and treatment of Arabidopsis with AIP shifted the interaction with Peronospora parasitica towards susceptibility. The low protection of Plasmopara viticola with BTH, an un- 
clear expression pattern of $P R-1$, and the weak effect on resistance of AIP alone rules out any major involvement of the SA signaling pathway.

LOX inhibitors have been successfully used to inhibit wound- or elicitor-induced responses in various plants (EllardIvey and Douglas 1996; Pena-Cortes et al. 1993; Staswick et al. 1991). Rickauer and associates (1997) investigated the role of LOX and jasmonate in tobacco plants infected with Phytophthora parasitica var. nicotianae by using the LOX inhibitor ETYA. Aziz and associates (2003) observed that laminarin did not induce cell death in grapevine, but increased defenserelated gene expression (including $L O X-9$ ), phytoalexin (resveratrol and viniferin) production, and activity of chitinases and $\beta$-1,3-glucanases. At the same time, laminarin induced protection against $B$. cinerea and Plasmopara viticola. In our study, the LOX inhibitor ETYA was combined with BABA. ETYA itself had no toxic effect on $P$. viticola but could reduce the JA-dependent responses of grapevine. The BABA-IR in Chasselas and the basal resistance of Solaris decreased with co-application of BABA and ETYA. The partial protection against $P$. viticola by JA, the potentiated expression of marker genes of the JA pathway, and the observed suppression of resistance by ETYA strengthen the argument of an involvement of the JA signal transduction pathway in BABA-IR against $P$. viticola.

In conclusion, the present study shows that BABA induced resistance against $P$. viticola in both susceptible and resistant cultivars of grapevine. The resistance in BABA-treated Chasselas plants and the basal resistance in Solaris depended to a large extent on the deposition of callose, which was positively correlated with BABA- and JA-induced resistance. LOX-9 and $P R-4$ gene expression was potentiated in BABA-treated plants upon infection with $P$. viticola. BABA-IR was significantly suppressed by the LOX inhibitor (ETYA) rather than by the PAL inhibitor (AIP). BTH and ABA failed to trigger induced resistance against $P$. viticola. On the other hand, the synergistic effect of AIP with DDG strengthens the hypothesis that lignification and accumulation of phytoalexins contribute, in addition to callose deposition, to the expression of resistance. Our results suggest that primed callose deposition plays a major role in BABA-IR of grapevine and is largely dependent on the JA signal transduction pathway. Ongoing studies on the involvement of phytoalexins and stilbene synthases will give a clearer picture of BABA-IR in grapevine against downy mildew.

\section{MATERIALS AND METHODS}

\section{Biological materials.}

Plants were raised from cuttings. Both grape cultivars (Chasselas and Solaris) and an isolate of $P$. viticola (NCCR1) were obtained from RAC, Nyon, Switzerland. Seedlings were grown from stratified seeds (Chasselas) kindly provided by Syngenta, Switzerland. The seeds were planted in vermiculite at $30^{\circ} \mathrm{C}$ for germination. After 10 to 15 days, the seedlings were transferred to potting soil (Substrat 1, Klassman-Deilmann $\mathrm{GmbH}$, Germany) and kept at $25^{\circ} \mathrm{C}$ (16 h of light). Seedlings at the three- to five-leaf stage were used in the experiments. $P$. viticola was maintained on detached leaves of grapevine, seedlings, or whole potted plants by repeated inoculations at $20^{\circ} \mathrm{C}$.

\section{Leaf disk assay.}

The third and fourth leaves from the top of plants were used. Leaf disks (1 cm in diameter) were punched from different plants and disks were mixed. Leaf disks were inoculated with sporangia of $P$. viticola $\left(5 \times 10^{4}\right.$ sporangia $\left.\mathrm{ml}^{-1}\right)$. Inoculated leaf disks were stained with Lactophenol-trypan blue (Keogh et al. 1980) at different time points (1, 2, 3, 4, 5, and 6 dpi). The stained samples were examined with a light microscope.

\section{Treatment}

with BABA, BTH, JA, DDG, AIP, ETYA, and ABA.

BABA, JA, the callose synthase inhibitor DDG, the LOX inhibitor ETYA, and ABA were purchased from Sigma (Zwijndrecht, The Netherlands). The PAL inhibitor AIP was a gift from N. Amrhein, ETHZ, Zürich, Switzerland and the SA analog BTH was supplied by Syngenta, Basel, Switzerland.

The chemicals were dissolved in water (ETYA and ABA were dissolved in a few drops of ethanol prior to dissolving in water) at the indicated concentrations. Leaf disks were floated (lower surface uppermost) on the chemical solutions for $24 \mathrm{~h}$ in the dark. In the case of ETYA, the disks were floated for $2 \mathrm{~h}$ on the solution before the next treatment. The leaf disks were washed one time in water and then transferred to petri dishes containing three moist Whatman filter papers (16 disks/petri dish). Controls consisted of disks from nontreated or noninoculated plants. The leaf disks (lower surface uppermost) were inoculated with a sporangial suspension of $P$. viticola $(5$ $\times 10^{4}$ sporangia $\mathrm{ml}^{-1} ; 2 \times 10 \mu \mathrm{l}$ drops/disk). The petri dishes were sealed with parafilm (to maintain high relative humidity) and placed in a $20^{\circ} \mathrm{C}$ growth cabinet (16 h of light) for the rest of the time.

\section{Staining for microscopy.}

Staining with lactophenol-trypan blue. To observe the structures of $P$. viticola, inoculated leaf disks were stained with Lactophenol-trypan blue according to Keogh and associates (1980). The disks were incubated for $48 \mathrm{~h}$ in the staining solution. The staining solution was removed and the stained disks were destained with chloral hydrate $\left(2.5 \mathrm{~g} \mathrm{ml}^{-1}\right)$. Destained disks were mounted in chloral hydrate solution and examined with a light microscope. Hyphae, sporangiophores, and sporangia were stained blue.

Histochemical examination and quantification of callose deposition. Leaf disks was stained with decolorized aniline blue (water blue; Fluka, Buchs, Switzerland) for callose according to Smith and McCully (1978) and counterstained with $0.002 \%$ calcofluor (fluorescent brightener; Sigma, St. Louis) to visualize oomycete structures (Zimmerli et al. 2000). The disks were stored in $0.07 \mathrm{M}$ Na-phosphate buffer and examined with UV light using an epifluorescence microscope (BP 340-380 nm, LP $425 \mathrm{~nm}$ ). Callose deposition in response to $P$. viticola was quantified according to Ton and Mauch-Mani (2004).

Lignification. Inoculated leaf disks were boiled for $10 \mathrm{~min}$ in $95 \%$ ethanol and then treated with phloroglucinol $(10 \mathrm{~g}$ in $95 \mathrm{ml}$ of absolute ethanol) for $3 \mathrm{~min}$. The disks were washed in $25 \% \mathrm{HCl}$ (Cohen et al. 1999; Dai et al. 1995), mounted in glycerol (75\%), and examined with a light microscope.

\section{Induced resistance bioassays.}

Leaf disks (16 disks/treatment, 4 disks/replication, four replications/treatment) were treated with water (control), BABA, BTH, JA, DDG, AIP, and ABA in different combinations $24 \mathrm{~h}$ prior to challenge inoculation with sporangia of $P$. viticola $(5 \times$ $10^{4}$ sporangia/ml). The disks were placed in a $20^{\circ} \mathrm{C}$ growth cabinet (16 h of light). The number of sporangia per leaf disk were counted 6 dpi.

Seedlings of grapevine (Chasselas) were taken at the four- to five-leaf stage and placed in trays. A petri dish was placed at the bottom of each pot. The plants were soil drenched with $\operatorname{BABA}(0.5,1,2$, or $4 \mathrm{mM})$. Four holes were punched into the soil around the base of seedlings in each pot. Different concentrations of BABA solution were poured into the holes in every 
treatment. The plants were placed in a $20^{\circ} \mathrm{C}$ growth cabinet $(16$ $\mathrm{h}$ of light) for 2 days. Then the plants were inoculated by dipping the leaves into a sporangial suspension of $P$. viticola $(5 \times$ $10^{4}$ sporangia $\mathrm{ml}^{-1}$ ). The inoculated leaves were marked with a felt pen. Inoculated seedlings were covered with a moist polythene sheet (to ensure sufficient humidity) and placed in the same growth cabinet. The seedlings were watered by adding water to the petri dish when needed. The seedlings were observed to assess the infection (percent) and disease severity at 6 and 10 dpi. Disease intensity was visually assessed using a 1 -to- 5 scale, in which $1=$ no symptoms visible, $2=$ up to $25 \%$, $3=25$ to $50 \%, 4=50$ to $75 \%$, and $5=>75 \%$ of the leaf area is affected 6 and 10 dpi. Values presented in different graphs are means \pm standard error of the mean. Different letters indicate statistically significant differences (Tamhane's test ; $\alpha=0.05$ ).

\section{RNA gel blot analysis.}

Total RNA was extracted by homogenizing frozen leaf tissue in extraction buffer ( $2 \%$ cetyltrimethylammonium bromide, $2 \%$ polyvinylpyrrolidone K30, $100 \mathrm{mM}$ Tris-HCL, $\mathrm{pH} 8.0,25$ mM EDTA, $2 \mathrm{M} \mathrm{NaCl}$, spermine at $0.5 \mathrm{~g} \mathrm{liter}^{-1}$, and leaf tissue at $5 \mathrm{ml} \mathrm{g}^{-1}$ ). The homogenates were extracted twice with chloroform and the RNA was precipitated using $\mathrm{LiCl}$, as described by Sambrook and associates (1989). For RNA gel blot analysis, $10 \mu \mathrm{g}$ of total RNA were separated on a $1.2 \%$ agarose gel containing 5\% formaldehyde, blotted onto $\mathrm{Hy}$ bond-XL membranes (Amersham Biosciences, Buckinghamshire, U.K.) by capillary transfer. The RNA gel blot was hybridized at $65^{\circ} \mathrm{C}$ with cDNA probes labeled with ${ }^{32} \mathrm{P}$-dCTP by random primer labeling. Blots were exposed for $24 \mathrm{~h}$ on XOmat AR film (Kodak).

\section{ACKNOWLEDGMENTS}

This project was funded by the National Center of Competence in Research (NCCR) Plant Survival, a research program of the Swiss National Science Foundation. We thank Recherche Agricole de Changins, Nyon, Switzerland, for supplying grapevine plants; J. Ton for inspiring scientific discussions; N. Amrhein for the kind gift of AIP; and B. Gsell, Syngenta, for supplying us with seed of grapevine.

\section{LITERATURE CITED}

Anderson, P. J., Badruzsaufari, E., Schenk, P. M., Manners, J. M., Desmond, O. J., Ehlert, C., Maclean, D. J., Ebert, P. R., and Kazan, K. 2004. Antagonistic interaction between abscisic acid and jasmonate-ethylene signaling pathways modulates defense gene expression and disease resistance in Arabidopsis. Plant Cell 16:3460-3479.

Audenaert, K., Meyer, G. D., and Höfte, M. 2002. Abscisic acid determines basal susceptibility of tomato to Botrytis cinerea and suppresses salicylic acid-dependent signaling mechanisms. Plant Physiol. 128:492-501.

Aziz, A., Heyraud, A., and Lambert, B. 2004. Oligogalacturonide signal transduction, induction of related-related responses and protection of grapevine against Botrytis cinerea. Planta 218:767-774.

Aziz, A., Poinssot, B., Daire, X., Adrian, M., Bezier, A., Lambert, B., Joubert, J. M., and Pugin, A. 2003. Laminarin elicits defense responses in grapevine and induces protection against Botrytis cinerea and Plasmopara viticola. Mol. Plant-Microbe Interact. 16:1118-1128.

Bleeker, A. B., Estelle, M. A., Summerville, C., and Kende, H. 1988. Insensitivity to ethylene conferred by a dominant mutation in Arabidopsis thaliana. Science 241:1086-1089.

Busam, G., Junghanns, K. T., Kneusel, R. E., Kassemeyer, H. H., and Matern, U. 1997a. Characterization and expression of caffeoyl-coenzyme A 3-O-methyltransferase proposed for the induced resistance of Vitis vinifera L. Plant Physiol. 115:1039-1048.

Busam, G., Kassemeyer, H. H., and Matern, U. 1997b. Differential expression of chitinases in Vitis vinifera L. responding to systemic acquired resistance activators or fungal challenge. Plant Physiol. 115:1029-1038.

Cohen, Y. 1994. 3-Aminobutyric acid induces systemic resistance against Peronospora tabacina. Physiol. Mol. Plant Pathol. 44:273-288.

Cohen, Y. 2002. B-aminobutyric acid-induced resistance against plant pathogens. Plant Dis. 86:448-457.
Cohen, Y., and Gisi, U. 1994. Systemic translocation of ${ }^{14}$ C-DL-3-aminobutyric acid in tomato plants in relation to induced resistance against Phytophthora infestans. Physiol. Mol. Plant Pathol. 45:441-456.

Cohen, Y., Niderman, T., Mösinger, E., and Fluhr, R. 1994. Beta-aminobutyric acid induces the accumulation of pathogenesis related proteins in tomato (Lycopersicon esculentum L.) plants and resistance to late blight infection caused by Phytophthora infestans. Plant Physiol. 104:59-66.

Cohen, Y., Reuveni, M., and Baider, A. 1999. Local and systemic activity of BABA (DL-3-aminobutyric acid) against Plasmopara viticola in grapevines. Eur. J. Plant Pathol. 105:351-361.

Conrath, U., Pieterse, C. M. J., and Mauch-Mani, B. 2002. Priming in plant-pathogen interactions. Trends Plant Sci. 7:210-216.

Dai, G. H., Andary, C., Mondolot-Cosson, L., and Boubals, D. 1995. Histochemical studies on the interaction between three species of grapevine, Vitis vinifera, $V$. rupestris and $V$. rotundifolia and the downy fungus, Plasmopara viticola. Physiol. Mol. Plant Pathol. 46:177-188.

Delaney, T. P. 1997. Genetic Dissection of acquired resistance to disease. Plant Physiol. 113:5-12.

Duijff, B. J., Pouhair, D., Olivain, C., Alabouvette, C., and Lemanceau, P. 1998. Implication of systemic induced resistance in the suppression of Fusarium wilt of tomato by Pseudomonas fluorescens WCS417r and by nonpathogenic Fusarium oxysporum Fo47. Eur. J. Plant Pathol. 104:903-910

Ellard-Ivey, M., and Douglas, C. J. 1996. Role of jasmonate in the elicitorand wound-inducible expression of defence genes in parsley and transgenic tobacco. Plant Physiol. 112:183-192.

Farmer, E. E., Almeras, E., and Krishnamurthy, V. 2003. Jasmonates and related oxylipins in plant responses to pathogenesis and herbivory. Curr. Opin. Plant Biol. 6:372-378.

Flors, V., Ton, J., Jakab, G., and Mauch-Mani, B. Abscisic acid and callose: Team players in defense against pathogens? J. Phytopathol. 153:1-7.

Fournier, J., Pouenat, M. L., Rickauer, M., Rabinovitch-Chable, H., Rigaud, M., and Esquerre-Tugaye, M. T. 1993. Purification and characterization of elicitor-induced lipoxygenase in tobacco cells. Plant J. 3:63-70.

Gindro, K., Pezet, R., and Viret, O. 2003. Histological study of the responses of two Vitis vinifera cultivars (resistant and susceptible) to Plasmopara viticola infections. Plant Physiol. Biochem. 41:846-853.

Jakab, G., Cottier, V., Toquin, V., Rigoli, G., Zimmerli, L., Metraux, J. P., and Mauch-Mani, B. 2001. Beta-aminobutyric acid-induced resistance in plants. Eur. J. Plant Pathol. 107:29-37.

Keogh, R. C., Deverall, B. J., and McLeod, S. 1980. Comparison of histological and physiological responses to Phakopsora pachyrhizi in resistant and susceptible soybean. Trans. Br. Mycol. Soc. 74:329-333.

Kessmann, H., Staub, T., Hofmann, C., Maetzke, T., Herzog, J., Ward, E., Uknes, S., and Ryals, J. 1994. Induction of systemic acquired resistance in plants by chemicals. Annu. Rev. Phytopathol. 32:439-459.

Kortekamp, R., Wind, R., and Zyprian, E. 1997. The role of callose deposits during infection of two downy mildew-tolerant and two susceptible Vitis cultivars. Vitis 36:103-104.

Lu, J., Zhang, S. Q., Guao, X., Outlaw, W. H., Jr. 2001. ABA changes in response to Pierce's disease of grapevines. Page 7 in: Proceeding of the 52nd annual meeting. American Society for Enology and Viticulture (ASEV). San Diego, CA, U.S.A.

Mauch, F., Mauch-Mani, B., and Boller, T. 1988. Antifungal hydrolases in pea tissue II. Inhibition of fungal growth by combinations of chitinase and b-1,3-glucanase. Plant Physiol. 88:936-942.

Mauch-Mani, B., and Slusarenko, J. 1996. Production of salicylic acid precursors is a major function of phenylalanine ammonia-lyase in the resistance of Arabidopsis to Peronospora parasitica. Plant Cell 8:203212.

McConn, M., Creelman, R. A., Bell, E., Mullet, J. E., and Browse, J. 1997. Jasmonate is essential for insect defense in Arabidopsis. Proc. Natl. Acad. Sci. U.S.A. 94:5473-5477.

Melan, M. A., Dong, X., Endara, M. E., Davis, K. R., Ausubel, F. M., and Peterman, T. K. 1993. An Arabidopsis thaliana lipoxygenase gene can be induced by pathogens, abscisic acid and methyl jasmonate. Plant Physiol. 99S:220.

Omer, A. D., Thaler, J. S., Granett, J., and Karban, R. 2000. Jasmonic acid induced resistance in grapevines to a root and leaf feeder. J. Econ. Entomol. 93:840-845.

Pajot, E., Le Corre, D., and Silue, D. 2001. Phytograd and DL-beta-amino butyric acid (BABA) induce resistance to downy mildew (Bremia lactucae) in lettuce (Lactuca sativa L). Eur. J. Plant Pathol. 107:861-869.

Pena-Cortes, H., Albrecht, T., Prat, S., Weiler, E. W., and Willmitzer, L. 1993. Aspirin prevents wound-induced gene expression in tomato leaves by blocking jasmonic acid biosynthesis. Planta 191:123-128.

Pieterse, C. M. J., Vanwees, S. C. M., Vanpelt, J. A., Knoester, M., Laan, R., Gerrits, N., Weisbeek, P. J., and Van Loon, L. C. 1998. A novel signaling pathway controlling induced systemic resistance in Arabidopsis. Plant Cell 10:1571-1580. 
Rickauer, M., Bottin, A., and Esquerre-Tugaye, M. T. 1992. Regulation of proteinase inhibitor production in tobacco cells by fungal elicitors, hormonal factors and methyl jasmonate. Plant Physiol. Biochem. 30:579-584.

Rickauer, M., Brodschelm, W., Bottin, A., Veronesi, C., Grimal, H., and Esquerre-Tugaye, M. T. 1997. the jasmonate pathway is involved differentially in the regulation of different defence responses in tobacco cells. Planta 202:155-162.

Ryals, J. A., Neuenschwander, U. M., Willitis, M. G., Molina, A., Steiner, H., and Hunt, M. O. 1996. Systemic acquired resistance. Plant Cell 8:1809-1819.

Sambrook, J., Fritsch, E. F., and Maniatis, T. 1989. Molecular Cloning: A Laboratory Manual, 2nd ed. Cold Spring Harbor Laboratory Press, Cold Spring Harbor, NY, U.S.A Flors.

Silue, D., Pajot, E., and Cohen, Y. 2002. Induction of resistance to downy mildew (Peronospora parasitica) in cauliflower by DL-beta-amino- $n$ butanoic acid (BABA). Plant Pathol. 51:97-102.

Smith, M. M., and McCully, M. E. 1978. Enhancing aniline blue fluorescent staining of cell wall structures. Stain Technol. 53:79-85.

Staswick, P. E., Huang, J. F., and Rhee, Y. 1991. Nitrogen and methyl jasmonate induction of soybean vegetative storage protein genes. Plant Physiol. 96:130-136.

Staswick, P. E., Su, W. P., and Howell, S. H. 1992. Methyl jasmonate inhibition of root growth and induction of a leaf protein are decreased in an Arabidopsis thaliana mutant. Proc. Natl. Acad. Sci. U.S.A. 89:6837-6840.

Steiner, U., and Schönbeck, F. 1997. Induced resistance. Pages 272-297 in Resistance of Crop Plants Against Fungi. H. Hartleb, R. Heitefuss, and H. H. Hopp, eds. Gustav Fischer, Jena, Stuttgart, Lubeck, Ulm, Germany.

Sticher, L., Mauch-Mani, B., and Metraux, J. P. 1997. Systemic acquired resistance. Annu. Rev. Phytopathol. 35:235-270.

Thomma, B. P. H. J., Eggermont, K., Penninckx, I. A. M. A., MauchMani, B., Vogelsang, R., Cammue, B. P. A., and Broekaert, W. F.
1998. Separate jasmonate-dependent and salicylate-dependent defenseresponse pathways in Arabidopsis are essential for resistance to distinct microbial pathogens. Proc. Natl. Acad. Sci. U.S.A. 95:1510715111.

Ton, J., and Mauch-Mani B. 2004. $\beta$-Amino-butyric acid-induced resistance against necrotrophic pathogens is based on ABA-dependent priming for callose. Plant J. 38:119-130.

Ton, J., Jakab, G., Toquin, V., Flors, V., Iavicoli, A., Maeder, M. N. Métraux, J.-P., and Mauch-Mani, B. 2005. Dissecting the $\beta$-aminobutyric acid-induced priming phenomenon in Arabidopsis. Plant Cell 17:987-999.

Van Loon, L. C., Bakker, P. A. H. M., and Pieterse, C. M. J. 1998. Systemic resistance Induced by Rhizosphere Bacteria. Annu. Rev Phytopathol. 36:453-483.

Van Peer, R., Niemann, G. J., and Schippers, B. 1991. Induced resistance and phytoalexin accumulation in biological control of Fusarium wilt of carnation by Pseudomonas sp. strain WCS417r. Phytopathology 81:728-734.

Vijayan, P., Shockey, J., Levesque, C. A., Cook, R. J., and Browse, J. 1998. A role for jasmonate in pathogen defence of Arabidopsis. Proc. Natl. Acad. Sci. U.S.A. 95:7209-7214.

Xiong, L., Lee, H., Ishitani, M., and Zhu, J. K. 2002. Regulation of osmotic stress-responsive gene expression by the LOS6/ABA1 locus in Arabidopsis. J. Biol. Chem. 277:8588-8596.

Zimmerli, L., Jakab, G., Metraux, J. P., and Mauch-Mani, B. 2000. Potentiation of pathogen-specific defence mechanisms in Arabidopsis by beta-aminobutyric acid. Proc. Natl. Acad. Sci. U.S.A. 97:1292012925.

Zon, J., and Amrhein, N. 1992. Inhibitors of phenylalanine ammonialyase: 2-aminoindan-2-phosphonic acid and related compounds. Liebigs Ann. Chem. 6:625-628. 